\title{
Reflets
}

Revue ontaroise d'intervention sociale et communautaire

\section{L'économie sociale en Ontario français : analyse historique, pratiques actuelles et recherche de sens}

\section{David Welch}

Volume 5, numéro 1, printemps 1999

Pratiques et développement économique communautaire

URI : https://id.erudit.org/iderudit/026249ar

DOI : https://doi.org/10.7202/026249ar

Aller au sommaire du numéro

Éditeur(s)

Reflets : Revue ontaroise d'intervention sociale et communautaire

ISSN

1203-4576 (imprimé)

1712-8498 (numérique)

Découvrir la revue

Citer cet article

Welch, D. (1999). L'économie sociale en Ontario français : analyse historique, pratiques actuelles et recherche de sens. Reflets, 5(1), 54-74.

https://doi.org/10.7202/026249ar

Tous droits réservés (C) Reflets : Revue ontaroise d'intervention sociale et communautaire, 1999
Ce document est protégé par la loi sur le droit d'auteur. L'utilisation des services d'Érudit (y compris la reproduction) est assujettie à sa politique d'utilisation que vous pouvez consulter en ligne.

https://apropos.erudit.org/fr/usagers/politique-dutilisation/ 


\title{
L'économie sociale en Ontario français: analyse historique, pratiques actuelles et recherche de sens
}

\author{
David Welch \\ Professeur, École de service social, Université d'Ottawa
}

Le but de ce texte ${ }^{1}$ est de mettre en lumière certains éléments qui permettent de saisir la place de l'économie sociale au sein de la communauté franco-ontarienne. Dans un premier temps, nous brossons un tableau de certaines des pratiques sociales, comme le bénévolat, la vie associative, les organismes de bienveillance et les groupes communautaires du XIX ${ }^{\mathrm{e}}$ siècle jusqu'aux années 1990 , qui peuvent nous éclairer sur l'organisation sociale de la communauté franco-ontarienne. Ensuite, à partir de quelques pistes de réflexion, nous voulons mettre en lumière certains des acquis et des forces de la communauté franco-ontarienne susceptibles d'informer des formes possibles d'économie sociale au sein de cette communauté.

\section{L'organisation sociale de la communauté franco- ontarienne avant les années 1960}

\section{Le travail bénévole des femmes}

Les premiers exemples d'une organisation originale du social au sein de la communauté franco-ontarienne datent du début de la 
«Dès le début du siècle, les femmes commencent à s'associer afin d'offrir des services plus concertés pour le bienfait de diverses causes. Une des associations qui occupe une place marquante en Ontario français est la Fédération des femmes canadiennesfrançaises (FFCF) fondée en 1914 à Ottawa...» colonisation et se retrouvent en grande partie dans le travail bénévole des femmes, tant en milieu rural qu'urbain. Les femmes oeuvrent bénévolement comme sages-femmes ou comme infirmières, organisent des activités d'entraide communautaire et, à mesure que se développent diverses institutions socio-sanitaires dirigées par des communautés religieuses, elles y travaillent bénévolement, plus souvent qu'autrement.

Dès le début du siècle, les femmes commencent à s'associer afin d'offrir des services plus concertés pour le bienfait de diverses causes. Une des associations qui occupe une place marquante en Ontario français est la Fédération des femmes canadiennesfrançaises (FFCF) fondée en 1914 à Ottawa par Almanda WalkerMarchand (Brunet 1992). L'association prend un essor important alors que les femmes mettent sur pied des unités dans plusieurs paroisses de l'Ontario français, et ce jusqu'aux années 1960. D'abord créée pour apporter une assistance aux combattants de la Première Guerre mondiale (Brunet 1992), la FFCF diversifie ses oeuvres pour répondre aux besoins émergeants, s'occupant, entre autres, des victimes des désastres comme les grands feux de forêt dans le Nord-est ontarien en 1916 et en 1921. Dans ce contexte, les femmes organisent des activités de levée de fonds dans les paroisses et défendent en même temps les droits linguistiques et politiques des Franco-Ontariens et FrancoOntariennes. En 1923-24, la Fédération s'affilie au Conseil canadien de la sauvegarde à l'enfance et, en 1928, elle appuie l'amendement de l'Acte des jeunes délinquants à la conférence de la Cour juvénile du Canada (Desjardins 1991). Pendant les années 1930, Walker-Marchand et d'autres membres de la FFCF interviennent activement dans la lutte de la famille Dionne pour assumer la garde de leurs quintuplées (Welch 1997).

L'Union catholique des fermières de l'Ontario (UCFO), fondée en 1937, est aussi une association de femmes qui apporte une contribution importante en matière de services sociaux, particulièrement au sein des communautés rurales francophones. Tout comme la FFCF, l'UCFO s'inscrit dans la foulée des associations laïques étroitement liées à l'Église catholique, qui s'investissent dans un travail caritatif destiné à venir en aide aux plus démunis. 
Par contre, à l'exception des services sociaux gérés directement par la communauté franco-ontarienne, la majorité des services de type social qui se développent dans la province est unilingue anglophone. Très souvent ces divers services aux communautés sont gérés par des organismes privés à but non lucratif qui reçoivent des octrois du gouvernement provincial et des municipalités. Par exemple, depuis les années 1890, il existe des Sociétés d'aide à l'enfance, initialement regroupées sous la Children's Protection Act de 1893. Ces services demeurent assujettis aux lois provinciales mais sont financés surtout par les municipalités et la charité privée. Dans certains cas, la province verse des sommes additionnelles. Ainsi, il se développe en Ontario une mixité publique-privée, avec un rapport symbiotique entre les deux qui change au fil des années mais qui demeure présent (Valverde 1995).

\section{Les associations de bienveillance avec vocation sociale et économique}

La première Conférence de Saint-Vincent de Paul, créée en 1860, s'employait à distribuer des secours à domicile aux résidants de la Basse-ville d'Ottawa.En 1874, les membres de la Société faisaient la quête de viande chez les bouchers, de bois dans les scieries de la ville et chez les cultivateurs (Brault 1973:59-60). Les membres de cette société ont travaillé en collaboration avec les Soeurs de la Charité au cours de la crise financière de 1875 à 1880, finançant une cuisine pour les pauvres qui est aménagée et administrée par les religieuses (Brault 1942: 216). À la paroisse Sainte-Anne d'Ottawa, le curé Alleau fonda en 1875 une autre organisation, l'Oeuvre de la Saint-Martin, pour faire face à cette période de crise, et offrir des services aux paroissiens sans travail. L'organisme cherche à identifier les emplois disponibles et à faciliter les liens entre les employeurs et les personnes en quête d'emploi (Brault 1973 :60). On remarque déjà avec ces organismes, concentrés en milieu urbain et dirigés davantage par des hommes, des liens plus évidents entre l'économie et le social.

Au début de la Crise des années 1930, l'élite franco-ontarienne, regroupée autour de l'Association canadienne-française d'éducation de l'Ontario (ACFÉO), participe aux discussions 
"Jugeant le problème du chômage chez les jeunes plus pressant que celui du monde ouvrier en général, l'exécutif de l'ACFÉO suggère, en 1934, la transformation de l'Oeuvre des chômeurs en centre d'accueil catholique s'adressant spécifiquement aux jeunes. Il faut dire que c'est parmi cette population qu'on craint le plus l'influence du communisme...» devant mener à la création, dans le diocèse d'Ottawa, de l'Oeuvre des chômeurs. Organisé sous forme de projet pilote visant à encourager la création de centres semblables dans d'autres paroisses, le centre offrait du secours populaire, du logement pour les plus déshérités, ainsi que des conseillers spirituels et en main-d'oeuvre pour aider les chômeurs à se trouver du travail. Ce projet a duré sous diverses formes jusqu'en 1934 (Gravel 1980: 31). Jugeant le problème du chômage chez les jeunes plus pressant que celui du monde ouvrier en général, l'exécutif de l'ACFÉO suggère, en 1934, la transformation de l'Oeuvre des chômeurs en centre d'accueil catholique s'adressant spécifiquement aux jeunes. Il faut dire que c'est parmi cette population qu'on craint le plus l'influence du communisme (Gravel 1980:32).

L'ACFÉO songe aussi à cette époque à la création de centres récréatifs pour les jeunes francophones afin de contrer l'attrait des centres anglophones déjà organisés. Ce n'est par ailleurs qu'en 1957 que les religieux de Saint-Vincent-de-Paul fondent le Patro St-Vincent à Ottawa, un centre de loisirs s'adressant à l'époque uniquement aux jeunes garçons franco-ontariens.

\section{Les entreprises coopératives}

En plus des associations laïques regroupées surtout autour des paroisses, la communauté franco-ontarienne, soutenue et encouragée par ses élites politiques, religieuses et sociales organise déjà des coopératives au milieu du $19^{\mathrm{e}}$ siècle. Parmi les premières, on trouve des sociétés mutuelles, des formes de compagnies d'assurance à but non lucratif. La plus connue fut sans doute l'Union St-Joseph (maintenant l'Union du Canada) fondée en 1863 par des ouvriers canadiens-français dans l'Est ontarien et à Ottawa. Dans ses premières années, la Société ne comptait que 700 membres (Comeau 1982), mais entre 1895 et 1910, ce nombre augmente à 28000 en Ontario et au Québec. Les 8000 membres ontariens étaient regroupés en 145 conseils locaux avec des polices d'assurance couvrant 50000 à 60000 personnes de la communauté sur une population franco-ontarienne de 203000 . Avec son expansion, la direction est passée des mains de la classe ouvrière à celles de l'élite concentrée dans la région d'Ottawa (Comeau 
1982: 41-42). Il faut savoir qu'en 1970, le nombre de membres dépassait 50000 (Grimard et Vallières 1986). À noter cependant que malgré son esprit coopératif et l'objectif de rendre des services à but non lucratif, l'Union St-Joseph rejetait toute forme d'organisation socio-économique plus socialisante. Au contraire, tout en défendant les intérêts identitaires des Franco-Ontariens, l'Union St-Joseph s'oppose à toute intervention de l'État dans des questions sociales, percevant celle-ci comme faisant partie d'une menace socialiste ou communiste! Dans un livre publié en 1939, l'auteur Charles Leclerc écrit ainsi que:

... la Saint Joseph s'inscrit en faux contre les doctrines entachées de socialisme, qui demandent à l'État de se constituer en une sorte de providence, qui veulent faire peser sur tous les citoyens indifféremment le poids de la subsistance d'un certain nombre, qui veulent tuer l'initiative individuelle, et entraver la compétition...Plutôt que de verser, même modérément, dans les théories à saveur socialiste, elle rappelle que le Christ a dit qu'il $y$ aurait toujours des pauvres et que c'est eux qu'il a aimés (Leclerc dans Grimard et Vallières, 1986: 195).

"À peu près la moitié des caisses populaires qui existent aujourd'hui ont été fondées entre 1940 et 1949. C'est dans cette période que les Franco-Ontariens ont connu, souvent pour la première fois, une plus grande stabilité économique, leur permettant ainsi plus de possibilités d'épargne."
À beaucoup d'égards, on peut remarquer la même attitude en ce qui concerne la fondation des coopératives d'épargne en Ontario français. En effet, les Caisses populaires ont été fondées sur le même modèle que celles au Québec. Ceci est dû en grande partie à la présence d'Alphonse Desjardins à Ottawa;il travaillait comme sténographe de langue française à la Chambre des communes entre 1891 et 1916. Ainsi il a participé à la fondation des premières caisses populaires de la région, entre autres celles de Ste-Anne (1912) et de Notre Dame (1913) (Grimard etVallières 1986; Jean 1986; Bureau 1992). À peu près la moitié des caisses populaires qui existent aujourd'hui ont été fondées entre 1940 et 1949. C'est dans cette période que les Franco-Ontariens ont connu, souvent pour la première fois, une plus grande stabilité économique, leur permettant ainsi plus de possibilités d'épargne. La mise sur pied de cercles d'études par l'Union des cultivateurs franco-ontariens 
«Contrairement à la situation au Québec où l'État appuie davantage les diverses initiatives qui surgissent de la société civile, le gouvernement ontarien, dans les années 1930 et 1940, lié de près aux grands intérêts capitalistes, soutient beaucoup moins les projets coopératifs, encore moins ceux des Franco-Ontariens.» en milieu rural a certainement contribué à ce développement (Grimard et Vallières 1986; Jean 1986).

En 1940, le Centre social de l'Université d'Ottawa a été fondé comme centre d'éducation et d'activités contre tout ce qui était identifié comme «la menace communiste». À partir de son programme "Reconstruction sociale et économique», le Centre suggère que le mouvement coopératif serait un moyen essentiel pour permettre aux Franco-Ontariens de mettre sur pied leurs propres outils économiques. Grâce à ses cercles d'études et cours par correspondance, le Centre influence directement la mise sur pied de nouvelles caisses populaires en Ontario français (Bureau 1992).

Contrairement à la situation au Québec où l'État appuie davantage les diverses initiatives qui surgissent de la société civile, le gouvernement ontarien, dans les années 1930 et 1940, lié de près aux grands intérêts capitalistes, soutient beaucoup moins les projets coopératifs, encore moins ceux des Franco-Ontariens.Ainsi ces différents projets socio-économiques franco-ontariens se développent jusqu'à un certain point en marge des initiatives de la majorité, tant étatiques que privées. Malgré leurs limites, ces premières expériences demeurent pour les Franco-Ontariens des exemples démontrant comment ils peuvent se regrouper, créer et gérer des organismes à l'image de leur communauté.

L'étude de l'histoire socio-économique franco-ontarienne (Grimard et Vallières 1986; Welch 1994; 1995) suggère que cette communauté,jusqu'aux années 1930, pouvait être décrite comme ayant des activités socio-économiques mi-capitalistes, misurvivance; ces formes reflètent à la fois sa ruralité et l'influence prépondérante du clergé, des classes dirigeantes et des communautés religieuses. Les premières initiatives sociales reflètent ces mêmes réalités socio-économiques. Elles ont été marquées par la mobilisation sociale, entre autres de femmes, de cultivateurs et d'ouvriers. Ensemble ils ont fondé des associations d'entraide et de revendication et ont développé des structures institutionelles nouvelles (par exemple la formule coopérative) - le tout construit surtout à partir du travail bénévole. Certains de ces organismes, souvent avec une vision morale assez explicite, regroupaient aussi 
"Les changements socio-économiques des années trente et quarante ont transformé les visages économique et socioculturel de l'Ontario et ont eu un impact sur les diverses communautés francoontariennes.» des individus des classes moyennes soucieux d'aider ceux de leurs communautés vivant dans le besoin. Malgré les limites de leurs actions, leurs stratégies de solidarités locales assurent leur survie en dépit de nombreux changements dans une société en effervescence.

Les changements socio-économiques des années trente et quarante ont transformé les visages économique et socio-culturel de l'Ontario et ont eu un impact sur les diverses communautés franco-ontariennes. Pour faire face à ces bouleversements, les gens s'emploient à créer dans les villes, quand le nombre le permet, des institutions intermédiaires qui rappellent d'une certaine façon celles de la campagne. Par contre, dans bien des endroits, les jeunes francophones et leurs parents se voient forcés de fréquenter les institutions établies et gérées par la population anglophone dominante pour obtenir les services qu'ils ne peuvent se procurer en français.

\section{Les années 1960 : nouveaux visages de l'organisation socio-économique}

Les années 1960 représentent un virage historique important dans les positions de l'élite franco-ontarienne face à l'État. Traditionnellement, les élites religieuses et séculaires avaient confié à la famille et aux institutions religieuses, généralement francophones, la sauvegarde de l'identité franco-ontarienne. La sécularisation et la plus grande prise en charge des secteurs de la santé, des services sociaux et de l'éducation par l'État accentuent la «distanciation entre les élites traditionnelles et leur communauté» (Carrière 1993: 324). Dorénavant, les droits gagnés auprès du gouvernement provincial ou délégués par celui-ci (Welch 1988; Carrière 1993), déterminent en bonne partie les diverses formes de développement social au sein de la communauté franco-ontarienne.

Les pratiques sociales subissent des transformations influencées non seulement par l'interventionnisme grandissant de l'État, mais aussi par l'implication plus directe des associations et des groupes, 
"Actives depuis toujours dans le bénévolat au sein de la communauté, des Franco-Ontariennes commencent à construire leur propres solidarités axées sur leurs besoins et intérêts comme femmes et Franco-Ontariennes, plutôt que de continuer de se laisser exclure par le manque de volonté politique de la part de l'État ontarien...» surtout de femmes, et par la revendication des droits d'une communauté qui se reconnaît de plus en plus comme minoritaire à mesure que se développe une identité franco-ontarienne plutôt que canadienne française.

Actives depuis toujours dans le bénévolat au sein de la communauté, des Franco-Ontariennes commencent à construire leur propres solidarités axées sur leurs besoins et intérêts comme femmes et Franco-Ontariennes, plutôt que de continuer de se laisser exclure par le manque de volonté politique de la part de l'État ontarien; elles visent ainsi à fournir des services adéquats pour leurs communautés (Pelletier 1987). Abandonnant des organismes comme l'ACFO, encore dominé par des hommes, plusieurs femmes s'engagent dans un féminisme «axé principalement sur la sensibilisation des femmes à leurs besoins» (Cardinal 1992: 12). Certaines mettent sur pied de nouveaux groupes tels les Ontaroises de l'Est, Franco-femmes, dans le Nord de l'Ontario, et le Réseau des femmes du Sud (Cardinal et Coderre 1990b). D'autres travaillent à la mise sur pied de garderies, de foyers pour femmes victimes de violence, de services pour femmes immigrantes. Certains organismes féminins tels la Fédération nationale des femmes canadiennes-françaises (FNFCF) et l'Union culturelle franco-ontarienne (UCFO) qui existent depuis longtemps, reformulent leurs orientations en axant leurs pratiques sociales vers des questions telles la violence faite aux femmes (Cardinal et Coderre 1990b). Ces projets sont construits surtout à partir du travail bénévole mais, contrairement au passé, ces organismes sont financés, partiellement ou entièrement, par l'État en plus de constituer dans certains cas une source de création d'emplois, particulièrement pour les femmes. ${ }^{2}$

D'autre part, une minorité de personnes au sein de la communauté tente de susciter un questionnement plus radical sur la nature même des pratiques sociales. Actives aussi dans les luttes pour de meilleurs services en français, ces personnes interviennent à l'intérieur de groupes de femmes, d'artistes, de jeunes et d'une multitude d'organismes communautaires. Par leurs pratiques, elles cherchent à diminuer certains des écarts qui existent au sein de la communauté en ajoutant à la variable linguistique 
«Ces remises en question dans les années 1980 et 1990 facilitent le développement de nouvelles pratiques socio-économiques, telles la radio communautaire, les coopératives de logements, les garderies à but non lucratif, des projets d'alphabétisation populaire, des groupes d'entraide de tous genres, des organismes pour les communautés ethno-culturelles francophones et, plus récemment, le développement économique communautaire.» une analyse des classes, de sexes et des régions. Dans certains cas, elles gardent une distance face aux organismes plus traditionnels ou conservateurs comme les caisses populaires qui sont vus comme des défenseurs du statu quo socio-économique et peu démocratiques dans leur fonctionnement.

Ces remises en question dans les années 1980 et 1990 facilitent le développement de nouvelles pratiques socio-économiques, telles la radio communautaire, les coopératives de logements, les garderies à but non lucratif, des projets d'alphabétisation populaire, des groupes d'entraide de tous genres, des organismes pour les communautés ethno-culturelles francophones et, plus récemment, le développement économique communautaire. Ces pratiques cherchent à comprendre comment les secteurs à but non lucratif, le secteur public et jusqu'à un certain point le secteur privé, peuvent collaborer afin de développer de nouveaux projets socio-économiques.

L'Union des cultivateurs franco-ontariens (UCFO), fondée comme une association de cultivateurs de langue française en 1929, offre un bel exemple de cette nouvelle approche. Sa transformation s'opère en 1984 quand elle a acheté le journal AGRICOM (fondé par deux jeunes femmes d'Alexandria) pour aider à briser l'isolement des cultivateurs franco-ontariens et pour servir d'outil pour le développement socio-économique dans l'Ontario francophone rural. Le journal compte présentement 5 300 abonnés dont 1200 au Québec. Dans le cadre de la loi de 1993 sur l'inscription des entreprises agricoles et le financement des organisations agricoles, l'UCFO est maintenant reconnue comme le seul groupe représentant les cultivateurs francoontariens, ce qui lui permet de recevoir des cotisations pour financer son fonctionnement, ses projets et le journal AGRICOM à $20 \%$. En 1989, l'UCFO fonda les deux premiers Groupements de gestion agricole de l'Ontario dans l'Est de l'Ontario et en 1993 un autre dans le Nord-Est de l'Ontario. Co-financés par le gouvernement à $50 \%$ et par les cultivateurs eux-mêmes à $50 \%$, ces collectifs permettent à chaque groupe de 35 familles agricoles d'embaucher leurs propres spécialistes en gestion agricole (agroéconomistes) pour les aider dans l'exploitation et le développement de leur ferme. Moins dispendieux que les agro-économistes 
"Ces exemples de développements dans l'organisation socioéconomique de l'agriculture francoontarienne démontrent qu'il est possible de mieux utiliser les fonds gouvernementaux, d'assurer une plus grande démocratie et une responsabilité accrue à ceux et celles qui utilisent les services, et de respecter la langue et les besoins culturels de la communauté.» gouvernementaux liés au ministère de l'Agriculture, les agronomes travaillant directement pour les cultivateurs sont redevables aux familles agricoles elles-mêmes et non au gouvernement.

Au début de 1996, le gouvernement Harris, dans ses plans de décentralisation du ministère de l'Agriculture à Guelph, propose de subordonner les trois collèges agricoles régionaux à l'Université de Guelph. À son tour,l'UCFO propose un partenariat particulier entre le Collège d'Alfred et l'Université de Guelph ainsi que la gestion du Collège par un conseil autonome de directeurs et de directrices nommés par la communauté, totalement indépendant du ministère de l'Agriculture. Ces options, qui n'avaient pas été envisagées jusque-là, sont acceptées par le gouvernement provincial. Ces exemples de développements dans l'organisation socio-économique de l'agriculture franco-ontarienne démontrent qu'il est possible de mieux utiliser les fonds gouvernementaux, d'assurer une plus grande démocratie et une responsabilité accrue à ceux et celles qui utilisent les services, et de respecter la langue et les besoins culturels de la communauté.

Dans d'autres régions de la province, les Franco-Ontariens et les Franco-Ontariennes se donnent des défis identiques. À Hearst, Parmi elles, un groupe de femmes, fonde en 1982 une pépinière nommée la Maison Verte, organisée sous forme de coopérative de femmes. Né de quelques fonds du gouvernement fédéral, le projet fournit, en 1996, huit emplois à temps complet et 35 à temps partiel. Les participantes au projet font maintenant pousser des millions d'arbres, des plantes potagères et construisent actuellement de nouvelles serres pour amorcer la culture des tomates pour le marché local.

Ces quelques exemples démontrent que les institutions socioéconomiques et communautaires franco-ontariennes répondent au défi d'assurer de nouvelles formes de services et de création d'emplois. Se basant sur des initiatives communautaires, tout en reconnaissant l'importance du financement gouvernemental, elles essaient d'éviter la surbureaucratisation des organismes étatiques. En même temps, elles ont à faire face au défi d'éviter, au sein de leurs propres organisations, la tendance à devenir elles aussi bureaucratiques, trop professionnelles et antidémocratiques. 
"...il importe que les nouvelles formes de pratiques sociales et communautaires évitent le repli sur soi, ce qui mènerait à d'autres formes d'intolérance et d'exclusion et qu'elles privilégient plutôt de nouvelles manières d'unir leurs efforts à ceux d'autres communautés qui ont des intérêts communs.»
Finalement, il importe que les nouvelles formes de pratiques sociales et communautaires évitent le repli sur soi, ce qui mènerait à d'autres formes d'intolérance et d'exclusion et qu'elles privilégient plutôt de nouvelles manières d'unir leurs efforts à ceux d'autres communautés qui ont des intérêts communs. Tout ceci s'inscrit dans un «struggle between very different values; the logic of competition versus the logic of community; the logic of machines and machine efficiency versus the logic of people trying to make a life for themselves and participate meaningfully in their society" (Menzies 1996:xv). En fin de compte la question qui se pose est la suivante: «[will] the local be an extension of global uniformity, or [will] the global be an extension of local diversity?» (Menzies 1996: 19).

\section{Place et forme actuelle du développement socio- économique en Ontario français}

D'abord il est nécessaire de situer ce questionnement dans le contexte de ce qu'on appelle généralement la crise de l'Étatprovidence. Le rôle de l'État comme acteur important dans le développement de la communauté franco-ontarienne est sérieusement remis en question depuis l'élection des Conservateurs en 1995. Présentement, nous sommes confrontés à une diminution des programmes sociaux, à une grande valorisation de la privatisation et à la déréglementation dans tous les secteurs. Pendant que les gouvernements sabrent dans leurs dépenses au nom de la guerre contre le déficit et que «la croissance sans emploi» du secteur privé domine, de larges secteurs de la population se trouvent exclus du marché du travail (Welch 1997).

\section{Construire des actions sur les acquis de la communauté}

Dans cette période de coupures massives, les Franco-Ontariens et Franco-Ontariennes peuvent compter sur plusieurs acquis développés au cours de leur histoire. Par exemple, les communautés 
"La globalisation de

l'économie et la concentration de la richesse contrastent avec la volonté des communautés de diverses régions de se prendre en main grâce au développement local...» franco-ontariennes continuent d'assumer leur régionalisme. On n'a qu'à penser à la prise en main par les travailleurs de la Spruce Falls à Kapuskasing, à laquelle les Franco-Ontariens et FrancoOntariennes ont participé activement. La lutte pour la sauvegarde de l'usine est un bel exemple d'une collaboration entre le tiers secteur (caisse populaire locale et les groupes communautaires), le secteur privé (TEMBEC) et l'État (qui a acheté la centrale hydro-électrique de la Kimberly Clark). La globalisation de l'économie et la concentration de la richesse contrastent avec la volonté des communautés de diverses régions de se prendre en main grâce au développement local et «de s'impliquer dans la recherche de solutions aux problèmes auxquels ils sont confrontés» (Aubry 1997:75). Dans un tel contexte, Laville (1997:112) suggère que «ces initiatives locales sont nées à partir de trois dynamiques distinctes: la préoccupation d'insérer dans l'emploi des personnes exclues du marché du travail, le souci de créer des activités dans des espaces urbains et ruraux «défavorisés», la prise en charge de réponses à de nouvelles demandes en impliquant les usagers concernés, en veillant à l'accessibilité des services, en particulier dans les services de proximité». Malgré des formes de pratiques très diversifiées, on remarque «la volonté de réconcilier initiative et solidarité, alors que ces deux valeurs ont longtemps été séparées: à l'économique, l'entreprise et au social, le partage» (Laville 1997: 112).

Dans un deuxième temps, les nombreuses luttes qu'ils ont dû livrer ayant affiné leur sens d'identité de solidarité collective (les 10000 personnes au ralliement pour sauvegarder l'hôpital Montfort d'Ottawa en 1997 en sont un exemple profond) les Franco-Ontariens et Franco-Ontariennes peuvent construire sur cette identité qui demeure «la base ou le réservoir de créativité» (Cournoyer 1997:44), permettant «la possibilité expérimentée et reconnue de se comprendre et donc d'agir de manière coordonnée. L'importance des cultures héritées n'est pas seulement sentimentale ou idéologique, mais bel et bien pratique, si l'on garde présent à l'esprit qu'une culture transporte des routines de sens et de comportement qui facilitent le vivre et l'agir ensemble» (Perret 1995: 315). 


\section{L'économie sociale: entre le privé et l'État}

«Par économie sociale, on entend l'univers des pratiques et des formes de mobilisation qui ne font partie ni de l'entreprise privée à but lucratif, ni des appareils de l'État au sens étroit du terme...»
Tout en proposant une analyse de l'espace que l'économie sociale pourrait occuper et occupe déjà au sein de la communauté francoontarienne, il est important de définir, dans le contexte des pratiques décrites ci-haut, les paramètres de l'économie sociale.

Par économie sociale, on entend l'univers des pratiques et des formes de mobilisation qui ne font partie ni de l'entreprise privée à but lucratif, ni des appareils de l'État au sens étroit du terme (Reading 1994; Kendal et Knapp 1995). Il s'agit d'organismes dont les activités marchandes et la réalisation d'un profit sont au plus des moyens de réaliser des objectifs de développement social qui échappent à la logique de marché. L'économie sociale ainsi définie s'apparente à d'autres concepts, tels ceux de tiers secteur (Gidron, Kramer et Salamon 1992; Wistow et al.1994; Salamon 1994; Kendall et Knapp 1995; Taylor 1995), de développement communautaire (Favreau et Lévesque 1995), de développement économique communautaire (Fontan 1992; Favreau et Ninacs 1992) et d'économie solidaire (Laville 1994).

L'utilisation d'expressions telles que "tiers secteur» ou "communautaire» a l'avantage d'attirer l'attention, comme nous avons voulu le faire dans cet article, sur une foule de pratiques et de formes de mobilisations sociales trop souvent oubliées ou négligées dans les milieux scientifiques et politiques. Nous croyons qu'il faut cependant éviter de tomber dans le piège contraire, qui consiste à traiter les différents «secteurs» comme s'il s'agissait de réalités tout à fait distinctes, isolées (Browne et Landry 1995; Browne 1996). En fait, ces différents «secteurs» se chevauchent au point qu'il est souvent difficile de démêler le "public» du "communautaire», particulièrement dans le contexte ontarien (Valverde 1995).

D'autres définitions ou synthèses de l'économie sociale ont été développées. Les caractéristiques de l'économie sociale proposées par Lemieux et Vaillancourt donnent encore plus de poids à la définition que nous proposons, en ce sens qu'elles mettent en valeur la complexité et la richesse du phénomène:

- Ces entités poursuivent des objectifs à la fois sociaux et économiques. 
- Ces entités, en tant que partie prenante de l'économie, produisent des biens, des services et du support à l'ensemble des citoyens et des citoyennes.

- Ces entités, en tant que partie prenante de l'économie sociale, recherchent la rentabilité économique et non pas un maximum de profits. Cet élément permet d'inclure les coopératives dans l'économie sociale, que l'on ait affaire à des coopératives d'usagers visant la participation des usagers ou des coopératives de production ou de travailleurs permettant la participation des salariés.

- Ces entités sont dotées de leurs structures démocratiques propres, à partir desquelles elles prennent les décisions favorisant la jonction entre la participation des salariés, celles des usagers et usagères et, le cas échéant, celle des citoyens et citoyennes de la communauté environnante.

- Ces entités recourent de façon importante au travail rémunéré; elles sont donc génératrices d'emplois.

- Ces réalités, en somme, sont des lieux de cimentation de la cohésion sociale et du lien social (Eme et Laville 1994; Favreau et Lévesque 1996; Lemieux etVaillancourt 1997: 8). ${ }^{3}$

Cet article vise ainsi à proposer que toute analyse de l'économie sociale en milieu minoritaire doit être examinée en lien avec l'analyse des organismes sociaux qui fonctionnent au sein des diverses communautés franco-ontariennes tant urbaines que

«La question

demeure : une «nouvelle économie sociale» ne pourraitelle pas tisser des liens entre le passé et le présent, l'urbain et le rural, tout autant qu'entre l'économique et le social?» rurales. La question demeure: une «nouvelle économie sociale» ne pourrait-elle pas tisser des liens entre le passé et le présent, l'urbain et le rural, tout autant qu'entre l'économique et le social?

Comme nous avons indiqué dans la première partie de ce texte, les organismes franco-ontariens ont changé de rôle, de nature et de fonctionnement au fils des ans. Historiquement les coopératives et certaines des oeuvres de bienfaisance, avec un rôle socio-économique, pouvaient s'insérer jusqu'à un certain point dans la définition que nous venons de donner de l'économie sociale. Par contre, ceci est moins clair pour le travail de bénévolat, un travail non payé mais pourtant si essentiel, fait surtout par les femmes. Dans un tel contexte historique, devrait-on élargir nos définitions de l'économie sociale afin d'englober des pratiques 
qui ne sont pas partie prenante de l'économie formelle mais qui y ajoutent des dimensions importantes?

Quand nous étudions la période de 1970 jusqu'aux années 1990 par exemple, il est plus facile d'identifier plusieurs organismes qui ont adopté un fonctionnement plus démocratique, des objectifs à la fois sociaux et économiques, qui produisent des biens et des services et qui sont une source d'emplois. Souvent ces nouveaux projets socio-économiques démontrent une capacité de s'adapter à des situations changeantes. Ainsi, même si la population francoontarienne a perdu certaines de ses concentrations géographiques, il existe des possibilités de créer des projets adaptés aux situations locales.

Avec le développement de l'État-providence, beaucoup de pratiques locales et d'organismes à but non lucratif se sont fait absorber ou supplanter par l'État. Ceci nous amène à proposer que l'histoire des divers organismes franco-ontariens qui s'insèrent dans une définition de l'économie sociale est inséparable du développement de l'État-providence en Ontario.

C'est à partir du questionnement sur le rôle changeant de l'État et la nécessité pour la communauté franco-ontarienne de s'organiser face aux nouvelles réalités socio-économiques que nous nous demandons comment les diverses communautés devraient développer de nouveaux projets socio-économiques qui s'insèrent dans notre définition de l'économie sociale, qui dépendent moins des fonds gouvernementaux et qui s'appuient plus sur les ressources

"Est-ce que certains projets socioéconomiques alternatifs sont moins vulnérables que d'autres aux coupures gouvernementales et jusqu'à quel point pourraient-ils demeurer relativement indépendants de l'aide étatique?» et la créativité communautaires. Est-ce que certains projets socioéconomiques alternatifs sont moins vulnérables que d'autres aux coupures gouvernementales et jusqu'à quel point pourraient-ils demeurer relativement indépendants de l'aide étatique? Finalement, quel rôle devrait jouer l'État dans le développement continu de la communauté franco-ontarienne?

Tout en reprochant au gouvernement d'être trop rigide, hiérarchique et impersonnel, il est quand même normal qu'on continue à défendre la notion qu'il appartient à l'État, par le biais du système de taxation, de fournir des moyens financiers socioéconomiques tels que définis démocratiquement par les diverses communautés, francophones ou autres. Plusieurs constatent que 
«Les francophones

minoritaires ayant

peut-être moins

l'habitude que la majorité de s'appuyer sur l'État (sauf dans le cas des élites) et ayant eu le réflexe, souvent par nécessité, de ne compter que sur leurs propres moyens, sont peut-être mieux placés pour promouvoir une démocratisation à partir des dispositifs de la société civile.» le secteur privé, axé sur le profit, ne serait pas intéressé par des projets peu rentables mais essentiels au développement des communautés. Plutôt, ils défendent l'idée que les fonds devraient être fournis en partie par l'État et transférés aux groupes voués au développement socio-économique des communautés. De nouveaux projets pourraient avoir «l'avantage de la qualité (flexibilité, proximité aux clients et clientes, capacité d'innover...) et de la quantité (coûts moindres)..." (Browne et Landry 1995 : 370). Ces projets pourraient mieux refléter également la langue et la culture des diverses communautés.

Les francophones minoritaires ayant peut-être moins l'habitude que la majorité de s'appuyer sur l'État (sauf dans le cas des élites) et ayant eu le réflexe, souvent par nécessité, de ne compter que sur leurs propres moyens, sont peut-être mieux placés pour promouvoir une démocratisation à partir des dispositifs de la société civile. C'est aussi une façon d'aller chercher et de «rassembler de nombreux acteurs qui s'étaient détournés de la politique «traditionnelle» pour s'orienter vers des actions locales, tout en étant conscients de leurs limites» (Laville 1997: 113).

Notre intérêt face au potentiel de l'économie sociale doit cependant s'accompagner d'une mise en garde: celle-ci n'est pas une panacée à tous les problèmes auxquels la communauté francoontarienne devra faire face dans les prochaines années. «L'enjeu est de définir un nouveau contrat social qui ne soit plus fondé sur le couple marché-assistance mais sur un renouvellement et une réconciliation des valeurs d'initiative et de solidarité» (Laville 1997:114).

\section{En guise de conclusion}

Nous pouvons constater que les différents projets socioéconomiques passés et actuels ont joué un rôle essentiel dans la sauvegarde et le développement des communautés francophones en Ontario. Historiquement, les fondateurs et fondatrices des premiers organismes socio-économiques en Ontario français contestaient très peu le statu quo socio-économique. Ils ont mis 
sur pied des pratiques et des ressources afin de s'entraider et d'aider les autres à combler des besoins essentiels: alimentation, habillement, logement, soins de santé, éducation, frais d'enterrement - mais aussi pour répondre à des préoccupations d'ordre social, d'identité culturelle, de reconnaissance et de communautarisation. Cela donne lieu à la création de réseaux d'organismes, à l'enracinement de pratiques, de coutumes et de traditions.

Avec les années 60 et une intervention plus présente de la part de l'État, beaucoup de Franco-Ontariens se sont tournés davantage vers celui-ci pour de l'assistance dans la création de nouveaux projets sociaux. Au cours des dernières années, dans le contexte de l'essoufflement de l'État-providence, d'autres ont cherché à travers leurs interventions une plus grande autonomie visà-vis l'État et un questionnement des assises socio-économiques de notre société.

C'est à partir de ces remises en question que certains FrancoOntariens et Franco-Ontariennes ont développé de nouvelles pratiques sociales qui peuvent s'insérer dans notre définition de l'économie sociale. Ces nouveaux projets sociaux, comme les nouvelles coopératives (radio communautaire, coopératives de production, nouveaux organismes en milieu agricole) ou communautaires (garderies, services spécifiques aux femmes) ont pu offrir de nouveaux lieux de démocratie. Les questions socioéconomiques ne sont pas seulement «des problèmes techniques à être résolus administrativement par des experts et expertes, au lieu d'être résolus à travers un processus démocratique par ceux et celles qui utilisent et fournissent le service " (Browne \& Landry 1995 : 111). Certains de ces projets ont plus ou moins contribué à la production, à la reproduction et parfois à la remise en question des rapports entre classes, nations, groupes ethniques, hommes et femmes.

Il existe toutefois le danger de vouloir créer de nouveaux projets socio-économiques plus humanitaires et plus près des vrais besoins de gens de nos communautés «sans remettre vraiment en question les rapports de propriété dans un monde où la part de lion de la richesse - et du pouvoir — est aux mains de quelques- 
uns. L'économie ne deviendra jamais vraiment solidaire tant que des espaces publics plus vastes ne viendront pas s'ajouter aux espaces publics de proximité, tant que les principes démocratiques et coopératifs ne dépasseront pas le tiers secteur pour envahir les secteurs publics et privés» (Browne 1997: 13).

\section{Bibliographie}

AUBRY, François (1997). «Quel rôle pour l'économie sociale?», Possibles, vol. 21, no 2, 65-81.

AUBRY, François et Jean CHAREST (1995). Développer l'économie solidaire, éléments d'orientation. Document déposé au Conseil confédéral de la CSN à Québec les 13, 14 et 15 septembre, Montréal, Québec, CSN, Service de recherche.

BRAULT, Lucien (1973). Sainte-Anne d'Ottawa: Cent ans d'histoire: 1873-1973, Ottawa, Ontario, Paroisse Sainte-Anne d'Ottawa.

BRAULT, Lucien (1942). Ottawa: capitale du Canada de son origine à nos jours, Ottawa, Ontario, Éditions de l'Université d'Ottawa.

BROWNE, Paul Leduc (1997). «La post-social-démocratie, ou la dialectique de l'économie sociale». Présentation au $8^{\mathrm{e}}$ congrès biennal sur la politique sociale canadienne, Université de Régina, Régina, Saskatchewan.

BROWNE, Paul Leduc (1996). Love in a Cold World? The Voluntary Sector in an Age of Cuts, Ottawa, Canadian Centre for Policy Alternatives.

BROWNE, Paul Leduc et Pierrette LANDRY (1995). The "Third Sector» and Employment.A report to the Department of Human Resources Development, Ottawa, Canadian Centre for Policy Alternatives.

BRUNET, Lucie (1992). Almanda Walker-Marchand (1868-1949): Une féministe franco- ontarienne de la première heure, Ottawa, Fédération nationale des femmes canadienne- françaises.

BUREAU, Brigitte (1992). Un passeport vers la liberté: Les caisses populaires de l'Ontario de 1912 à 1992, Ottawa, Le Mouvement des caisses populaires de l'Ontario.

CARDINAL, Linda (1992a). «Théoriser la double spécificité des Franco-Ontariennes», Marie-Luce Garceau (dir) Relevons le défi! Actes du colloque sur l'intervention féministe dans le Nord-Est de l'Ontario, Sudbury, Ontario, 177-188.

CARDINAL, Linda (1992b). «La recherche sur les femmes francophones vivant en milieu minoritaire: un questionnement sur le féminisme", Recherches féministes, vol. 5, no 1, 5-29.

CARDINAL, Linda et Cécile CODERRE (1991). «Éducation et identité :l'expérience des femmes francophones vivant en milieu minoritaire», Éducation et francophonie :femmes et éducation, vol. 19, no 3, 23-26.

CARDINAL, Linda et Cécile CODERRE (1990a). "Les francophones telles qu'elles sont: les Ontaroises et l'économie», La Revue du Nouvel-Ontario, 12,151-181.

CARDINAL, Linda et Cécile CODERRE (1990b). «Reconnaitre une histoire : le mouvement des femmes francophones hors Québec», Femmes d'action, vol.19, no 3, 15-16.

CARRIÈRE, Fernand (1993). «La métamorphose de la communauté franco-ontarienne, 19601985», Cornelius J.Jaenan (dir) Les Franco-Ontariens, Ottawa, Ontario, Les Presses de l'Université d'Ottawa, 305-340. 
COMEAU, Gayle (1982). The Role of the Union Saint-Joseph du Canada in the Organization of the Association canadienne-française d'éducation d'Ontario, Montréal, Québec, Université de Montréal.

COURNOYER, Monique (1997). «Le travail: quelle crise?», Possibles, vol. 21, no 2, 29-46.

DESJARDINS, Micheline (1991). Les femmes de la diaspora canadienne-française. Brève histoire de la FNFCF de 1914 à 1991, Ottawa, Fédération nationale des femmes canadiennes-françaises.

EME, Bernard et Jean-Louis LAVILLE (s. la dir. de) (1994). Cohésion sociale et emploi, Paris, Desclée de Brouwer.

FAVREAU, L. et B. LÉVESQUE (1996). Développement économique communautaire, économie sociale et intervention, Sillery, Presses de l'Université du Québec, Collection pratiques et politiques sociales.

FAVREAU, L. et B. LÉVESQUE (1995). Repenser le développement communautaire et l'économie sociale à la faveur de la crise de l'emploi, Montréal, Québec, Cahiers de CRISES.

FAVREAU, L. et W. A. NINACS (1992). «Le développement économique local et communautaire au Québec», Coopératives et développement, vol. 23, no 2, 115-123.

FONTAN, Jean-Marc (1992). «La démocratie économique communautaire», Possibles, vol. 16, no 1, 53-64.

GIDRON, Benjamin, Ralph M. KRAMER et Lester M. SALAMON (1992). «Government and the Third Sector in Comparative Perspective: Allies or Adversaries?», Benjamin Gidron, Ralph M. Kramer et Lester M. Salamon (s.la dir. de) Government and the Third Sector: Emerging Relationships in Welfare States, San Francisco, California, Jossey-Bass.

GRAVEL, Jacques (1980). Quelques aspects de la vie des Franco-Ontariens durant les années de la Grande Dépression (1930-1939), Toronto, Ontario, Université York.

GRIMARD, Jacques et Gaétan VALLIÈRES (1986). Travailleurs et gens d'affaires canadiens-français en Ontario, Montréal, Éditions Études Vivantes.

JEAN, Sylvie (1986). Histoire du mouvement coopératif en Ontario français. Ottawa, Ontario, Conseil de la coopération d'Ontario.

JUTEAU-LEE, Danièle et Barbara ROBERTS (1981). «Ethnicity and Femininity: (d') après nos expériences", Revue canadienne des études ethniques, vol. 8, no 1,1-23.

JUTEAU-LEE, Danièle (1983). «Ontarois et Québécois: Relations hors-frontières?», Dean R. Louder et Eric Waddel (dir.) Du continent perdu à l'archipel retrouvé: le Québec et l'Amérique française, Québec, Les Presses de l'Université Laval, 42-53.

KENDALL, J. et M. KNAPP (1995). A Loose and Baggy Monster: Boundaries, Definitions and Typologies, J. D. Smith, C. Rochester et R. Hedley (s. la dir. de) An Introduction to the Volunteer Sector, Londres et New York, Routledge, 66-95.

LABELLE, Marielle (1984). La reproduction et l'appropriation dans des organismes de femmes francophones dans le nord de l'Ontario : 1850 à 1950, thèse de maitrise, Université d'Ottawa.

LAVILLE, Jean-Louis (1997). «Un nouveau contrat social», Possibles, vol. 21, no 2, 106-114.

LAVILLE, Jean-Louis (1994). «Sociologie économique», L'économie solidaire : une perspective internationale, Paris, Desclée de Brouwer.

LECLERC, Charles (1939). L'Union Saint-Joseph du Canada, son histoire, son oeuvre, ses artisans! Ottawa, Ontario.

LEMIEUX, Diane et Yves VAILLANCOURT (1997). L'économie sociale et la transformation du réseau des services sociaux et de santé: définitions, état de la situation et enjeux, Montréal, Laboratoire de recherche sur les pratiques et les politiques sociales. 
LÉVESQUE, Benoît (1997). L'économie sociale, dérision ou panacée?, Montréal, Conseil québécois de développement social, 5-10.

LÉVESQUE, Benoit et Marie-Claire MALO (1992). «L'économie sociale au Québec: une notion méconnue, une réalité économique importante», Jacques Defourny et Jose-Luis Monzon (s. la dir. de) Économie sociale, entre économie capitaliste et économie publique / The Third Sector , Bruxelles, Centre international de recherches et d'information sur l'économie publique, sociale et coopérative, De Boeck University, 173-192.

MELUCCI, Alberto (1993). «Vie quotidienne, besoins individuels et action volontaire», Sociologie et Sociétés, vol. 25, no 1,189-197.

MENZIES, Heather (1996). Whose Brave New World?, Toronto, Between the Lines.

PELLETIER, Jacqueline (1987). Discours d'ouverture, Sudbury, Rencontre des féministes francoontariennes, non publié.

PELLETIER, Jacqueline (1980). «Les Franco-Ontariennes», Les cahiers de la femme, vol. 2, no 2, 6063.

PERRET, B (1995). L'avenir du travail. Les démocraties face au chômage, Paris, Seuil.

PROULX, P. (1982). La part des femmes, il faut la dire, Ottawa, La Fédération des femmes canadiennesfrançaises.

RACINE, Sonia et Lisa VERREAULT (1997). «Synthèse des échanges», L'économie sociale. Dérision ou panacée?, Montréal, Conseil québécois de développement social, 30-33.

READING, Paul (1994). Community Care and the Voluntary Sector. The Role of Voluntary Organisations in a Changing World, Birmingham, Venture Press/British Association of Social Workers.

SALAMON, Lester (1994). «The Rise of the Nonprofit Sector», Foreign Affairs, July/August.

TAYLOR, M. (1995). «Volunteer Action and the State», David Gladstone (s. la dir de.) British Social Welfare. Past, Present and Future, Londres, ULC Press, 140-214.

VALVERDE, Mariana (1995). «The Mixed Social Economy as a Canadian Tradition», Studies in Political Economy, 47, 33-60.

WELCH, David (1997). «The Franco-Ontarian Community Under the Harris counter- Revolution», Diana Ralph,André Régimbald and Nérée St-Amand Open for Business / Closed to people, Halifax, Fernwood Publishing, 122-133.

WELCH, David (1995). «Les Franco-Ontariens : la résistance comme mode de vie», Reflets, vol. 1, no $1,20-42$.

WELCH, David (1994). «Formes socio-économiques et identité franco-ontarienne dans le nord-est de l'Ontario", Égalité, no 34, automne, 46-80.

WELCH, David (1988). The Social Construction of Franco-Ontarian Interests Towards French-language Schooling, $19^{\text {th }}$ Century to 1980 s, Thèse de doctorat inédite, Université de Toronto.

WISTOW, G., M. KNAPP, B. HARDY et C. ALLEN (1994). Social Care in a Mixed Economy, Buckingham and Philiadelphia, Open University Press. 


\section{Notes}

1. Ce texte s'appuie sur des recherches en cours avec Paul Leduc Browne, du Centre canadien en politiques alternatives. La section sur les services sociaux et services de santé est tirée d'une recherche non publiée faite conjointement avec Madeleine Dubois de l'École de Service Social, Université d'Ottawa. Cette recherche a bénéficié de soutien financier de la part du Développement des ressources humaines Canada (DRHC).

2. Certains des écrits qui cherchent à conceptualiser les FrancoOntariennes comme sujet sont: Cardinal (1992a, 1992b); Cardinal et Coderre (1990a, 1990b, 1991); (Coderre 1995); Juteau-Lee (1983), Juteau- Lee et Robarts (1981) Labelle (1984), Pelletier (1980,1987); Proulx (1982).Voir aussi la revue féministe Le tablier déposé, publiée dans l'Est ontarien entre 1979-1985.

3. Voir aussi le Comité de travail sur l'économie sociale dans Aubry (1997) et Racine et Verreault (1997:33) pour d'autres définitions. 